\title{
Lamb wave assessment of fatigue and thermal damage in composites
}

\author{
Michael D. Seale ${ }^{\mathrm{a})}$ \\ Department of Physics, The College of William and Mary, Williamsburg, Virginia 23187
}

Barry T. Smith

Norfolk Academy, 1585 Wesleyan Drive, Norfolk, Virginia 23502

W. H. Prosser

NASA Langley Research Center, Mail Stop 231, Hampton, Virginia 23681

(Received 13 August 1997; accepted for publication 28 January 1998)

\begin{abstract}
Among the various techniques available, ultrasonic Lamb waves offer a convenient method of evaluating composite materials. Since the Lamb wave velocity depends on the elastic properties of a structure, an effective tool exists to monitor damage in composites by measuring the velocity of these waves. Lamb wave measurements can propagate over long distances and are sensitive to the desired in-plane elastic properties of the material. This paper describes two studies which monitor fatigue damage and two studies which monitor thermal damage in composites using Lamb waves. In the fatigue studies, the Lamb wave velocity is compared to modulus measurements obtained using strain gage measurements in the first experiment and the velocity is monitored along with the crack density in the second. In the thermal damage studies, one examines samples which were exposed to varying temperatures for a three minute duration and the second includes rapid thermal damage in composites by intense laser beams. In all studies, the Lamb wave velocity is demonstrated to be an excellent method to monitor damage in composites. (C) 1998 Acoustical Society of America. [S0001-4966(98)02705-2]
\end{abstract}

PACS numbers: 43.35.Zc, 43.40.Le, 43.20.Mv [HEB]

\section{INTRODUCTION}

Composite materials are being more widely used today by aerospace, automotive, sports equipment, and a number of other commercial industries because of their advantages over conventional metals. Composites have a high strength-toweight ratio and can be constructed to meet specific design needs. In addition to satisfying the design specifications, composites offer weight savings over traditional metals without sacrificing strength. With the advantages of composite materials, their use is continually increasing and becoming more widespread to a variety of fields. ${ }^{1-3}$

O'Brien et al. ${ }^{4}$ and Charewicz and Daniel ${ }^{5}$ have examined the fatigue behavior of laminates and proposed lifetime prediction models based on fatigue factors such as increased matrix cracking, delamination growth, and stiffness loss. Ogin et al.$^{6,7}$ and Lim and Hong ${ }^{8}$ have modeled the stiffness reduction in composites due to the effects of matrix cracking. Laws and Dvorak ${ }^{9}$ have investigated the progression of matrix cracking as well as stiffness loss due to transverse cracking.

In addition to fatigue damage, thermal degradation of composites is of concern. Street et al. ${ }^{10}$ have examined how composites respond to thermal damage of temperatures up to $350{ }^{\circ} \mathrm{C}$ for short durations, as well as exposure times as long as $30 \mathrm{~min}$ for temperatures of $225^{\circ} \mathrm{C}$. Herakovich and Hyer $^{11}$ and Ermer and Mall ${ }^{12}$ have studied the effects on composites which were thermally cycled. The temperature

\footnotetext{
a) Current address: NASA Langley Research Center, Mail Stop 231, Hampton, VA 23681.
}

range used was $-157{ }^{\circ} \mathrm{C}-+121^{\circ} \mathrm{C}$ in Ref. 11 and $149^{\circ} \mathrm{C}-$ $427^{\circ} \mathrm{C}$ in Ref. 12. Stansfield and Pritchard ${ }^{13}$ have subjected composites to rapid changes in temperature. These fluctuations, similar to the flight profile of supersonic aircraft, can lead to moisture absorption and matrix cracking.

Traditional nondestructive evaluation (NDE) techniques used on metals are often not easily adaptable to composites due to the inhomogeneous and anisotropic nature of these materials. Finding an effective means of nondestructively monitoring damage in composites is a challenging task, but it is extremely important to the safety and reliability of such structures. Several techniques are available to nondestructively evaluate damage in materials. ${ }^{14-16}$ Salkind $^{14}$ and Stinchcomb ${ }^{15}$ detail various methods used for damage detection in composites such as ultrasonics, x-ray radiography, thermography, and holography. In addition, Daniel et al. ${ }^{16}$ examined the use of ultrasonic backscatter to monitor crack density and compared the results to those obtained using $\mathrm{x}$-ray radiography.

Among the various techniques available, ultrasonic Lamb waves offer a convenient method of evaluating these composite materials. As a material is damaged, the elastic parameters of the structure change. Since the Lamb wave velocity depends on these properties, an effective tool exists to monitor damage in composites by measuring the velocity of these waves. Additionally, Lamb wave measurements are better than conventional through-the-thickness ultrasonic measurements because they can propagate over long distances and are sensitive to the desired in-plane elastic properties of the material. 
Studies have been conducted which show a reduction in Lamb wave velocity due to a loss of stiffness caused by matrix cracking. ${ }^{17-19}$ All of the authors noted that the inplane properties of a composite are affected by cracking and that they can be monitored through the use of Lamb waves, which travel perpendicular to the crack direction. Dayal and Kinra $^{17}$ showed that for through-the-thickness measurements, the attenuation is sensitive to cracking, while the velocity is not. For Lamb wave propagation in the plane of the plate, both wave speed and attenuation were found to be sensitive to cracking. Tang and Henneke ${ }^{18}$ noted that Lamb waves provide information about the in-plane properties of a plate, and this type of measurement is more useful due to the fact that composites are commonly designed to carry inplane loads. Similarly, Dayal et al. ${ }^{19}$ noted that the Lamb wave interaction with cracks is much stronger in the plane of the plate and, thus, provides an effective method to detect damage due to transverse matrix cracking.

Given the ability to nondestructively monitor materials using guided waves, studies have been conducted which use Lamb waves to monitor a variety of material properties in composite structures. Karim et al. ${ }^{20}$ and Mal et al..$^{21}$ have used inversion techniques to determine the material parameters of composites from experimental Lamb wave data. Habeger $e t \mathrm{al}^{22}$ studied the use of plate waves to monitor the strength of paper for the purpose of monitoring quality control on line for the paper industry. Several authors ${ }^{23-25}$ have used Lamb waves to inspect adhesively bonded joints. Lamb wave techniques have also been used to study delaminations, ${ }^{26-29}$ porosity, ${ }^{29,30}$ and fiber misalignment. ${ }^{30}$ Recently, Lamb waves have even been used by Lee $e t$ al. ${ }^{31}$ to measure the temperature of silicon wafers during processing and by Pei et al. ${ }^{32}$ for in situ measurements of thin film thickness. Both of these techniques would be of interest to the semiconductor processing industry.

While numerous studies exist which examine fatigue damage in composites using nondestructive techniques, few, if any, have been conducted which monitor thermal damage using ultrasonic nondestructive evaluation techniques. In the studies mentioned earlier, ${ }^{10-13}$ the effects of thermal damage were measured using mainly mechanical tests and microscopic observation. This work will focused on the use of Lamb waves to nondestructively monitor fatigue as well as thermal damage in composite structures.

The following sections describe two experimental studies which monitor fatigue damage in composites using Lamb waves. In the first study, the Lamb wave velocity was compared to modulus measurements obtained using strain gage measurements. Additionally, experimental dispersion curves are compared for damaged and undamaged specimens. In the second study, the Lamb wave velocity was monitored along with the crack density. For both studies, the Lamb wave velocity measurements showed a significant decrease as the samples underwent increasing amounts of damage.

In addition to the fatigue studies, two studies were also conducted which monitor thermal damage in composites using the Lamb wave technique. The first study involves samples which were heated to varying temperatures for a three minute duration. The second study examined rapid thermal damage in composites. These samples were subjected to a very short duration $(0.5$ and $1.0 \mathrm{~s})$ laser source. In both studies, the Lamb wave velocity was demonstrated to be an excellent method to monitor thermal damage in composite materials.

\section{FATIGUE DAMAGE 1}

In this experiment, studies have been conducted which monitor fatigue damage in composite samples using strain gage measurements as well as Lamb wave velocity measurements. A description of the test samples is followed by the results of two different measurements of Lamb wave velocity. The first technique is a contact measurement done at a single frequency, while the second involved an immersion study of Lamb waves in which dispersion curves were obtained. The results of the Lamb wave method for monitoring fatigue damage are compared to the damage progression measured using strain gages.

The composite samples studied were AS4/3501-6 graphite/epoxy with a stacking sequence of $\left[0 / 90_{3}\right]_{S}$. Two 30.5 -by $38.1-\mathrm{cm}$ plates were manufactured and $\mathrm{C}$-scanned prior to being cut into specimens to check for any abnormalities. The scans revealed a 5\%-7\% level of porosity in the samples. The plates were then cut into 28 -by $3.8-\mathrm{cm}$ coupons. The finished coupons had an average thickness of 0.12 $\mathrm{cm}$. Two $0.635-\mathrm{cm}$ strain gages were attached to each sample: one axial and one transverse. From the values measured for the stress, axial strain, and transverse strain, one can obtain Young's modulus and Poisson's ratio for the sample.

Before the fatiguing process, two samples were loaded quasi-statically to failure to obtain Young's modulus, Poisson's ratio, and ultimate strength. Each of these coupons was loaded from 0 to 3250 microstrain and back twice. On the third run, the samples were loaded to failure. The modulus and Poisson's ratio were obtained by averaging the values measured for each of the three load cycles described above. The ultimate strength was defined as the load at which the samples failed.

The specimens were subjected to tension-tension fatigue in a 55-kip capacity load frame. The samples were fatigued at a frequency of $10 \mathrm{~Hz}$ and at an $R$ value (minimum load/maximum load) of 0.3 . The upper load was taken to be $33 \%$ of the ultimate strength, which had a value of 160 MPa. The specimens were successively raised to higher fatigue cycle values. The velocity of the lowest order symmetric Lamb mode was measured and the modulus obtained from strain gage measurements both before and after each cyclic loading. The samples were removed from the load frame at intermediate values of fatigue cycles in order to make the contact measurements.

Lamb modes can be generated by placing a longitudinal contact transducer on a plate and driving it with a sine wave tone burst. Ditri et al. ${ }^{33}$ showed that periodic loading normal to the surface of a plate, as produced by a longitudinal transducer, will excite Lamb waves at distances away from the source which are large compared to the wavelength of the Lamb mode. Lamb waves are generated in most materials at a distance of roughly $1 \mathrm{~cm}$ or less. Since measurements are 


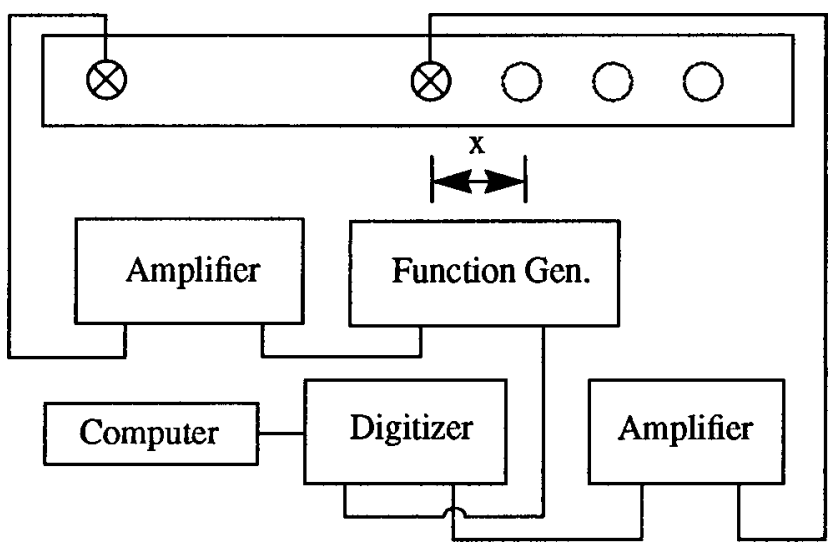

FIG. 1. Experimental arrangement for the contact measurement.

normally done anywhere from 5 to $10 \mathrm{~cm}$ away from the source, this does not create any difficulties in generating and measuring Lamb modes using this technique.

A Lamb wave was generated by using a 2-cycle, 0.5$\mathrm{MHz}$ sine wave to excite the sending transducer. The signal was received at various positions, digitized at $10 \mathrm{MHz}$, and the waveforms saved on a computer. Both the sending and receiving transducers were broadband longitudinal wave transducers with a nominal center frequency of $1.0 \mathrm{MHz}$ and a diameter of $1.27 \mathrm{~cm}$. A coupling gel was used between the transducers and the plate. The experimental arrangement is shown in Fig. 1.

The time differences were measured by imposing a computational delay on the first signal to overlap the signals received at greater distances. The small thickness of the plates combined with the low frequency used to generate the Lamb wave yield a frequency-thickness product of $0.6 \mathrm{MHz} \cdot \mathrm{mm}$. In this region, only the $S_{0}$ and $A_{0}$ modes propagate. Therefore, the leading part of the wave was identified as the lowest order symmetric Lamb wave, which is not very dispersive. The trailing portion of the signals contained the dispersive antisymmetric wave as well as edge reflections. The distances were measured to an accuracy of $0.01 \mathrm{~cm}$ using a sonic ranger. A least-squares fit from a plot of time and distance was performed to obtain the velocity of the prompt $S_{0}$ mode.

As the samples underwent increasing amounts of fatigue, an increasing number of cracks developed in the $90^{\circ}$ plies. These cracks degrade the modulus. Photomicrographs at different magnifications were taken of the edge of a fatigued composite and are shown in Fig. 2. For the lowest magnification, the fibers from the $0^{\circ}$ laminas are the vertical bright streaks on the outer edges and the six $90^{\circ}$ laminas are what appear as dots in between the outer layers. The ends of the fibers for these laminas is seen quite clearly at the higher magnification shown in Fig. 2(d). The size of the crack shown is approximately $1 \mu \mathrm{m}$.

The composite samples exhibited a decrease in the velocity as the modulus degraded due to increasing fatigue. Both the modulus and velocity squared follow the same general trend with increasing fatigue cycles. This is shown schematically in Fig. 3. In the plot, the velocity was normalized to the velocity measured before fatiguing. The normalized

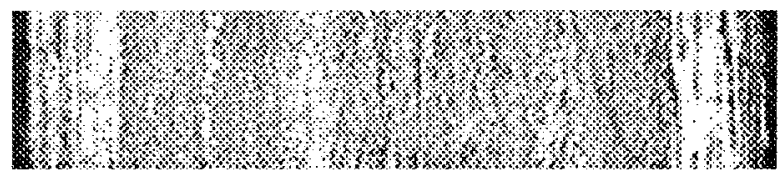

(a)

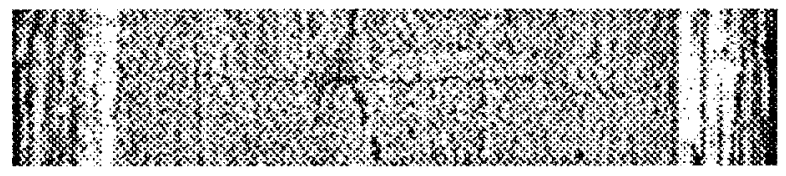

(b)

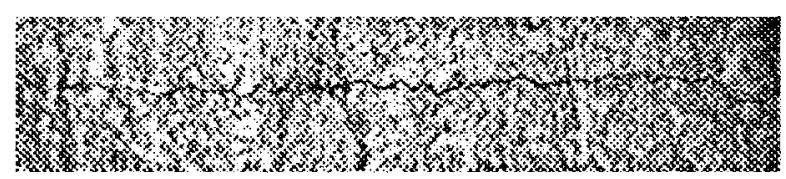

(c)

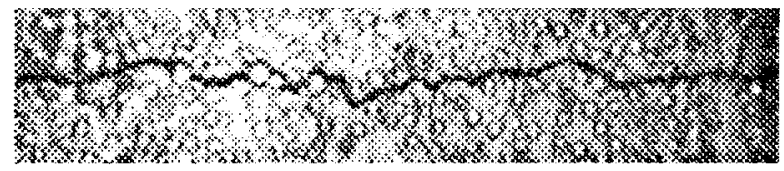

(d)

FIG. 2. Photomicrographs of the edge of a composite sample: (a) without a crack and (b)-(d) with a transverse matrix crack. The magnification for each was: (a) 5.5; (b) 5.5; (c) 11.0; and (d) 28.0. The thickness of the sample is $0.12 \mathrm{~cm}$.

modulus was obtained in a similar manner. The velocity squared error bars are an estimate of the total error due to the uncertainties in the distance measurement and the digital signal overlap used to obtain the time differences. Some of values for the three measurements on the individual samples varied as much as $8 \%-10 \%$ from the average. Using a similar technique, Tang and Henneke ${ }^{18}$ have reported deviations as great as $10 \%$ from the average of three measurements as well.

The velocity of the lowest order symmetric mode depends on the square root of the modulus for isotropic plates and the square root of the in-plane stiffness values for unidirectional composites. ${ }^{34}$ Since the modulus shown in the plot was obtained from strain gage measurements of the in-

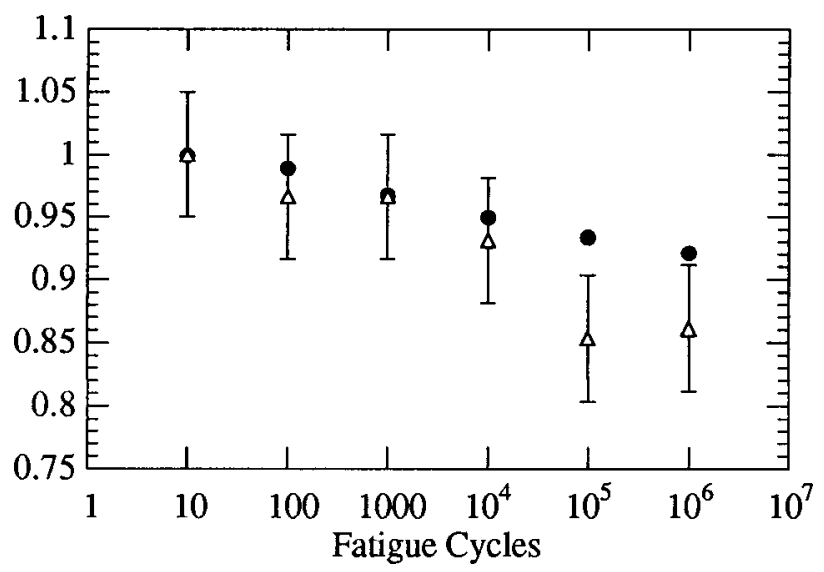

FIG. 3. Plot of normalized modulus (solid circles) and normalized velocity squared (open triangles) versus fatigue cycles for a fatigued sample. 


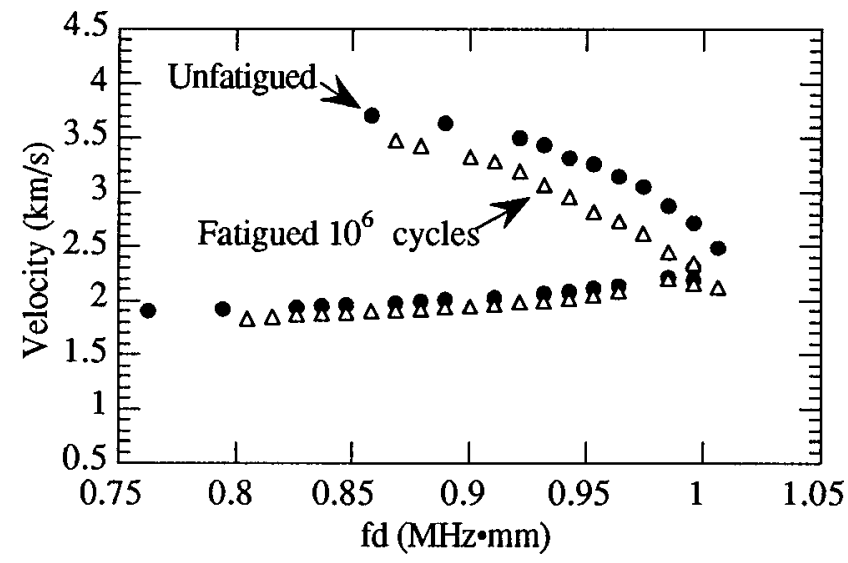

FIG. 4. Plot of the lower modes of the experimental dispersion curves for a sample which is undamaged (solid circles) and one which has been fatigued $10^{6}$ cycles (open triangles).

plane stiffness, the square of the velocity was chosen to plot against the number of fatigue cycles for convenience.

In addition to the contact measurements, which were done at a single frequency, an immersion method was used to obtain dispersion curves for two samples. The experimental arrangement for this type of measurement is detailed by Balasubramaniam et al. ${ }^{35}$ In this method, the sample was immersed in water, which serves as the couplant between the transducer and the plate, and the same transducer was used to generate and receive the ultrasonic signal. An aluminum block served as a reflector for the input pulse as the specimen was rotated about the corner. When a Lamb wave is excited in the plate, part of the total energy is converted to a propagating Lamb mode. Due to this loss of energy, a minimum in the frequency spectrum of the reflected signal corresponds to the frequency at which a Lamb mode is propagating.

The reflected waveform at each angle was saved for later analysis of the frequency content. The velocity can be determined from Snell's law using the fact that only a longitudinal wave is supported in water and that the Lamb mode is propagating in the plate. After determining the frequency of the minimum for each angle, the velocity verses the frequency-thickness product can be plotted to obtain the dispersion curve. The frequency range is determined by the bandwidth and frequency of the transducer used.

In this experiment, an immersion transducer with a nominal center frequency of $1.0 \mathrm{MHz}$ and diameter of 2.86 $\mathrm{cm}$ was used in a pulse-echo arrangement. The specimen was rotated $0.4^{\circ}-60^{\circ}$ in steps of $0.4^{\circ}$ and the reflected waveform at each angle was saved for later analysis of the frequency content. After determining the frequency of the minimum for each angle, the velocity (from Snell's law) verses the frequency-thickness product was plotted to obtain the dispersion curves. This technique was used to obtain dispersion curves for an undamaged sample as well as one which had one million fatigue cycles. The results are shown in Fig. 4.

The dispersion curves for the fatigued sample is clearly shifted from the undamaged sample for both of the lower modes. The curves have roughly a $7 \%$ difference in velocity for the symmetric mode. This is in good agreement with the

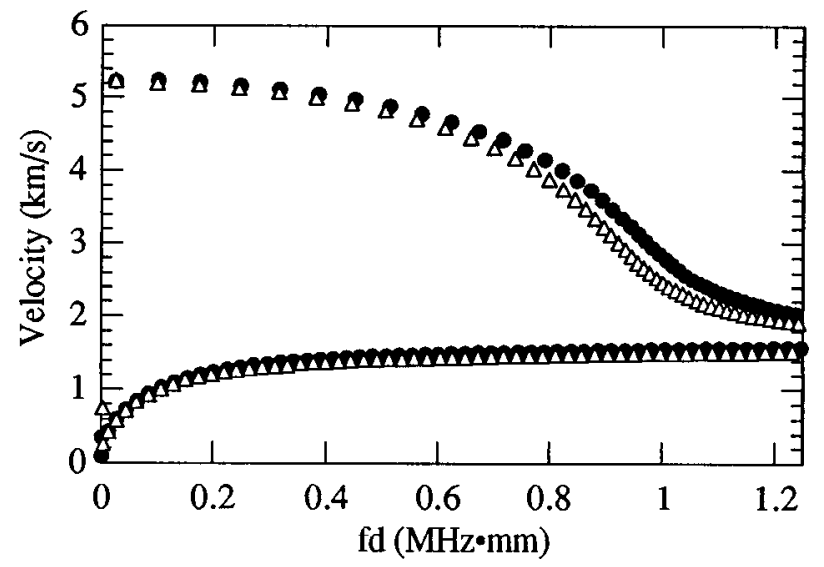

FIG. 5. Comparison of lower modes of numerical curves using full parameters (solid circles) and with $c_{33}$ reduced by $5 \%$ (open triangles).

contact result for the same sample, which had a $7.2 \%$ relative decrease in velocity after one million fatigue cycles. It is also noted in the figure that the $A_{0}$ mode velocity variation was much less than that of the $S_{0}$ mode. This can be understood by examining how the velocity relates to the modulus. The velocity changes as the square root of the stiffness for the symmetric mode, but as the fourth root of the stiffness for the antisymmetric mode. ${ }^{34}$ Therefore, the $A_{0}$ mode velocity will change less than the $S_{0}$ mode velocity for a given decrease in modulus.

For propagation across the fibers, where the 1-axis is in the fiber direction and the 3-axis is perpendicular to the fibers, a decrease in the elastic stiffness parameter $c_{33}$ alters the dispersion curves. This is due to the fact that $c_{33}$ is the modulus across the fibers. The transverse matrix cracks (due to fatigue damage) should, therefore, lead to a decrease in the elastic stiffness constant $c_{33}$. To examine the effect of the matrix cracking, a through-the-thickness finite element model, detailed in Dong and Huang ${ }^{36}$ and Datta et al., ${ }^{37}$ is used to generate dispersion curves in laminated composites. Numerical dispersion curves were calculated in which the value of $c_{33}$ was reduced by $5 \%$ in only these layers. A comparison was done for the numerical dispersion curves for the lower modes. The curves for the lower modes calculated using the full elastic parameters and those calculated using $c_{33}$ reduced by $5 \%$ in the $90^{\circ}$ layers are shown in Fig. 5 . When compared in the region where the experimental data was obtained $(0.75 \leqslant f d \leqslant 1.05 \mathrm{MHz} \cdot \mathrm{mm})$, the numerical results (see Fig. 5) are in good agreement with the experimental dispersion curves shown in Fig. 4. For the theoretical curves in this region, the $S_{0}$ velocity shows roughly a $7 \%$ decrease and the $A_{0}$ mode variation is much less than the $S_{0}$ velocity change. These are exactly the characteristics which were observed in the experimental curves.

It has been noted by Charewicz and Daniel ${ }^{5}$ that strain gages only measure a local change in modulus and do not measure a global change in the specimen. Since the strain gages used in this study had a gauge length of $0.635 \mathrm{~cm}$, they may not give an accurate account of damage throughout the entire plate. This would be especially true at the lower fatigue levels where very few cracks were observed in the samples. As the samples were damaged further, the cracking 
became more uniform. Thus a Lamb wave technique at low frequencies used to track the modulus as a function of fatigue history may provide improved global interrogation over the propagation length of the wave.

\section{FATIGUE DAMAGE 2}

Additional composite samples of the same material, AS4/3501-6, and same architecture, $\left[0 / 90_{3}\right]_{S}$, were obtained in order to conduct a second fatigue damage study. The new composites were loaded to greater levels in order to induce damage more quickly in the samples. Two composite plates identical to those used in the previous study were obtained. The new composite plates were C-scanned prior to cutting them into coupons to check for porosity. The images were very uniform and, therefore, no large scale areas of porosity were expected. The plates were then cut into 18 3.8-by 28$\mathrm{cm}$ coupons. The edges were polished and photomicrographs were taken. No appreciable amounts of porosity were found.

Similar to the first study, the specimens have been subjected to tension-tension fatigue. The samples were fatigued at a frequency of $10 \mathrm{~Hz}$ and at an $R$ value of 0.35 . For this study, however, the upper load was taken to be $45 \%$ of the ultimate strength, which has a value of $200 \mathrm{MPa}$. The samples were fatigued for a range of $10^{n}$ cycles $(n=3,4$, and 5 ). As before, the samples were removed from the load frame at intermediate values of fatigue cycles and the velocity of the lowest order symmetric Lamb mode was measured. In addition to loading these samples at higher levels, the crack density was also monitored during the process.

A Lamb wave was generated by using a 6-cycle, 0.5$\mathrm{MHz}$ sine wave to excite a longitudinal wave transducer with a nominal center frequency of $0.5 \mathrm{MHz}$ and a diameter of $1.27 \mathrm{~cm}$. The signal was received at various positions by an array of pinducers with active elements of $0.135 \mathrm{~cm}$. The pinducers were placed in a holder at a fixed separation distance of $1.0 \mathrm{~cm}$ between each transducer. This receiving arrangement greatly reduces the error in the measurement of the velocity due to the uncertainty in the distance between the transducers. Since a differential measurement is being made, fixing the transducers in a holder virtually eliminates the distance error. A coupling gel was used between the transducers and the plate.

The signals were digitized at $10 \mathrm{MHz}$, averaged, and the waveforms saved to the computer. In an effort to reduce the error incurred in the time measurement by human judgement of the overlap region, the time differences between the pinducers were measured by performing a cross correlation. The leading part of both waves, which is identified as the lowest order symmetric Lamb wave, was used in the correlation. The distances between the five pinducers in the array were held fixed at 1,2,3, and $4 \mathrm{~cm}$, respectively. The velocity of the prompt $S_{0}$ mode was obtained by a least-squares fit from a plot of time and distance.

To measure the crack density, a $2.5-\mathrm{cm}$ section in the middle of the plate was marked. This was done in order to count the number of cracks occurring in the same $2.5-\mathrm{cm}$ length in the sample. Keeping the same measurement area is especially important at the lower cycle levels where the cracking is not very uniform. The number of cracks was counted by examining the edges of the coupons under a microscope. The velocity measurements described above were centered over the same region where the crack density was monitored.

The influence of fatigue cycles on transverse matrix cracking and Lamb wave velocity was examined for five different samples. The velocity and crack density were obtained after each cyclic loading. Each velocity was obtained from the average of three measurements. The standard deviation for all of the samples was less than 5\% from the average of the three measurements and most were within $2.5 \%$ of the average for each individual sample. Thus the reproducibility of the measurements is quite good and provides an improvement over the technique used in the first fatigue study. Also, these results are similar to those obtained by Tang and Henneke, ${ }^{18}$ who report a variation of $1.5 \%$ using a similar technique.

As expected, the crack density for these samples increased with increasing cycles and, as with the previous study, the velocity squared decreased with fatigue cycles. Since strain gages were not used on these samples, the decrease in modulus will be estimated from the crack density. An expression which relates reduced modulus to crack density is given in Caslini et al. ${ }^{38}$ For a $\left[0_{m} / 90_{n}\right]_{S}$ laminate, the stiffness loss is given by ${ }^{38}$

$$
\frac{E}{E_{0}}=\left[1+\frac{n E_{2}}{m E_{1}} \frac{\tanh (\lambda / 2 D)}{(\lambda / 2 D)}\right]^{-1},
$$

where

$$
\lambda^{2}=\frac{3 G_{12} E_{0}}{h^{2} E_{1} E_{2}} \frac{(n+m)}{n^{2} m} .
$$

In the above equations, $E$ and $E_{0}$ are the values of the modulus for the damaged and undamaged laminate, respectively. $E_{1}, E_{2}$, and $G_{12}$ are the moduli (longitudinal, transverse, and shear, respectively) for each individual unidirectional lamina in the plate. $D$ is the crack density, $h$ is the lamina thickness, and $m$ and $n$ are the number of $0^{\circ}$ and $90^{\circ}$ layers, respectively, in the half-thickness of the plate.

For the case presented here, $m=1, n=3$, and $h$ $=0.15 \mathrm{~mm}$. The value for the undamaged modulus, $E_{0}$, was obtained from the average of the four modulus measurements for the samples which were loaded to failure. The engineering constants for AS4/3501-6, are taken to be ${ }^{1}$

$$
E_{1}=142.0, E_{2}=10.34, G_{12}=7.17,
$$

where all values are listed in GPa.

The reduced stiffness as a function of crack density was calculated from the parameters given above using Eqs. (1) and (2). Figure 6 shows a comparison of the calculated reduced stiffness and the normalized velocity squared for all of the samples in this study. The velocity was normalized in a similar manner as described previously. Since the uncertainty in the distance and time measurements in this study is less than that of the earlier study, the velocity error bars are not as large as were previously shown. Due to the possibility of overlooking a small crack under the microscope, the error in calculation of the crack density is assumed to be a \pm 1 error in the total number of cracks counted in the $2.5-\mathrm{cm}$ 


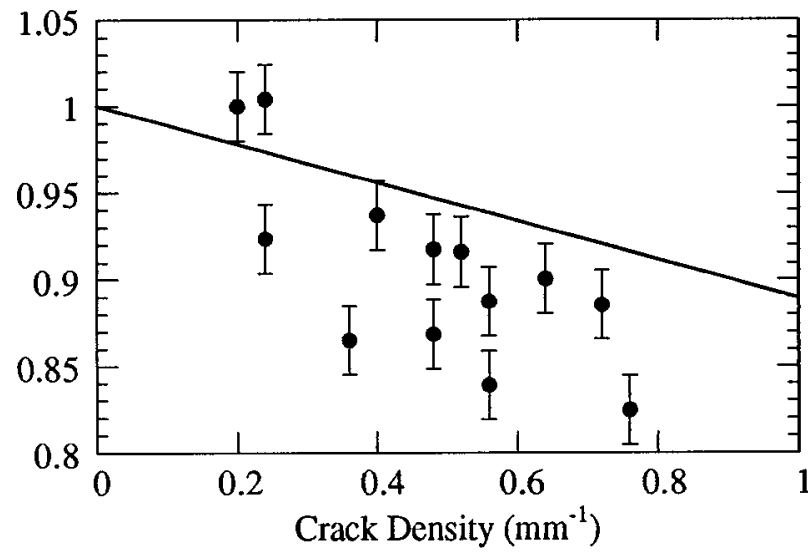

FIG. 6. Calculated normalized modulus (solid line) versus normalized velocity squared (solid circles) as a function of crack density for all samples.

length. However, for clarity, the error bars for the crack density have been omitted in the figure. Figure 6 shows a larger decrease for the normalized velocity squared than for the normalized modulus which was calculated from the crack density. Although not as pronounced as the results shown in Fig. 6, this behavior was evident in the previous study when the modulus was measured using strain gages (see Fig. 3).

These results are also in agreement with those found by Dayal et al. ${ }^{19}$ who examined the modulus degradation of an AS4/3502, $\left[0 / 90_{3}\right]_{S}$ composite using velocity measurement of the lowest order symmetric Lamb mode. The architecture and the fibers (AS4) of the samples used by Dayal et al. ${ }^{19}$ are identical to the ones studied here. The epoxy matrix used (3502) is slightly different than the one used here, but the material properties of the two samples are very close.

Similar to the measurements done here, the tests by Dayal et al. ${ }^{19}$ were also done at a frequency of $0.5 \mathrm{MHz}$. The velocity of the $S_{0}$ mode, measured using a different technique, and the crack density, measured using edge replication, were monitored as the samples underwent increasing damage. The modulus was calculated directly from the velocity measurement using the relation ${ }^{19}$

$$
E=\rho \nu^{2},
$$

where $E$ is the modulus, $\rho$ is the density, and $\nu$ is the velocity of the $S_{0}$ Lamb mode. Given this relation, the normalized modulus will also be equal to the normalized velocity squared. The results from Dayal et al. ${ }^{19}$ are presented, along with the results for a representative sample from this study, in Fig. 7.

In the figure, the solid line represents the theoretical reduced modulus previously calculated using the material parameters for AS4/3501-6. However, as mention earlier, the parameters for this type of composite are extremely close to those of AS4/3502. Therefore, the theoretical curve shown in the figure is representative of the composites used in both studies. Another interesting feature in the figure involves the comparison of the two velocity measurements. Both sets of data show a larger decrease in normalized velocity squared than what is theoretically predicted for the modulus reduction. Dayal et al. ${ }^{19}$ reported similar results when the modulus reduction measured using the Lamb wave technique was

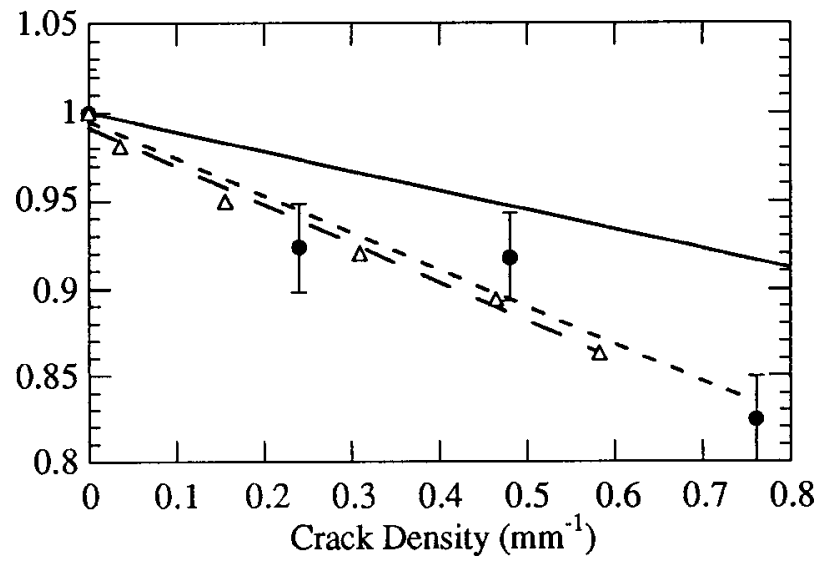

FIG. 7. A plot of the normalized velocity squared for a fatigued sample (solid circles) versus data from Dayal et al. (Ref. 19) (open triangles). Curve fits are shown for the data in this study (short dashes) and for the data of Dayal et al. (Ref. 19) (long dashes). The solid line is the theoretical modulus reduction described in the text.

compared with the results of analytical modeling and static stiffness measurements conducted by other authors. Thus the Lamb wave velocity technique may provide a more sensitive method of damage detection than modulus reduction measurements.

Presented in the second fatigue study are the results of a different technique than that which was used in the previous study for determining the Lamb wave velocity in composites. Due to a reduction in the uncertainty in the distance and time measurements, the error in the velocity measurements were found to be less using this technique than those found in the first study. The crack density was also monitored as a function of fatigue cycles. A decrease in normalized velocity squared with increasing fatigue cycles was noted. This was expected and agrees with the results presented in the initial study.

The main difference between this study and the initial fatigue study was the monitoring of the crack density with fatigue cycles. The crack density was observed to increase with an increasing number of cycles, as is to be expected, and the Lamb wave velocity exhibited a decrease with increasing crack density. This drop in velocity as a function of crack density was compared with the results of Dayal et al., ${ }^{19}$ who saw a similar velocity decrease.

As was mentioned before, strain gages only measure a local change in modulus and do not measure a global change in the specimen. The strain gages used in the previous study had a gauge length of $0.635 \mathrm{~cm}$ and therefore, may not have given an accurate account of damage throughout the entire plate. It was shown in Caslini et al. ${ }^{38}$ that the crack density can be theoretically related to stiffness reduction. Since the crack density was monitored over a $2.5-\mathrm{cm}$ length, this becomes the effective gauge length for measuring the modulus reduction via the crack density.

The modulus reduction, calculated from the crack density, was compared to the results obtained using the Lamb wave velocity measurements. A more dramatic decrease was observed in the velocity measurements than in the theoretical modulus reduction. Thus a Lamb wave technique used to track damage in composites may provide improved interro- 


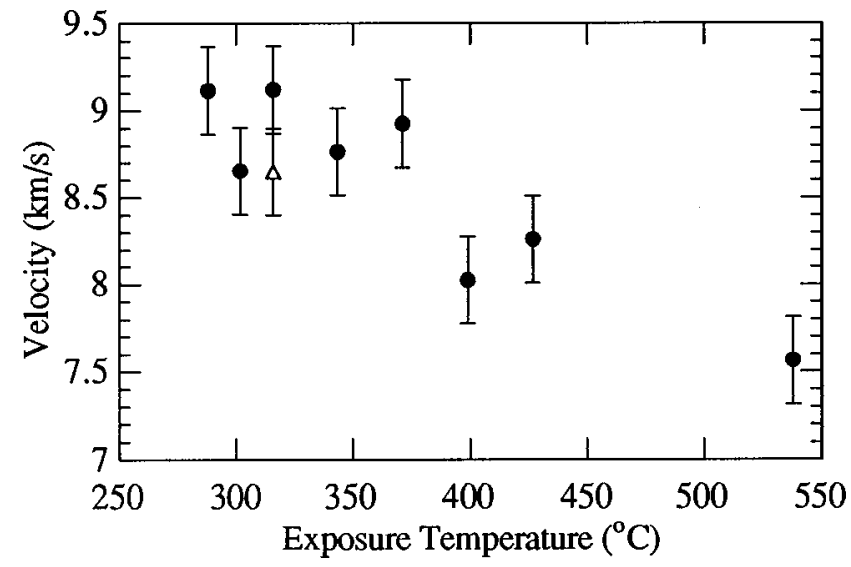

FIG. 8. Plot of $S_{0}$ velocity for a range of samples exposed to different temperatures for durations of $3 \mathrm{~min}$ (solid circles) and $10 \mathrm{~min}$ (open triangles).

gation of the effective stiffness of a structure over the propagation length of the wave.

\section{THERMAL DAMAGE}

Determining the response of composites to thermal damage is necessary for a complete understanding of the total use environment of these materials. Heat damaged samples, which simulate typical thermal damage on carbon/epoxy aircraft structures, were provided by McDonnell Douglas Aerospace. These 15.24-by $15.24-\mathrm{cm}$ specimens were fabricated as 16-ply, uniaxial plates of AS4/977-3 and had an average thickness of $3.07 \mathrm{~mm}$. All samples had received different levels of heat damage, had been C-scanned, and had Barcol hardness readings performed. The exposure temperatures ranged from $288{ }^{\circ} \mathrm{C}$ to $538{ }^{\circ} \mathrm{C}$ for a duration of $3 \mathrm{~min}$ and included a sample which was exposed to $316^{\circ} \mathrm{C}$ for $10 \mathrm{~min}$.

Lamb waves were generated and received with the same techniques described in the second fatigue study. The only differences were that the transmitting transducer had a nominal center frequency of $1.0 \mathrm{MHz}$ and the receiving transducers had fixed separations of $4,5,6$, and $7 \mathrm{~cm}$. The signal was received at various positions and digitized at $10 \mathrm{MHz}$. The time differences were measured by imposing a computational delay on the first signal to overlap the signals received at greater distances. A least-squares fit from a plot of time and distance was performed to obtain the velocity of the prompt $S_{0}$ mode.

As in the fatigue studies, the velocity of the lowest order symmetric Lamb mode was measured. The results of the velocity measurements in the fiber direction for ten different thermally damaged samples are plotted in Fig. 8. The error bars are an estimate in the velocity error and are mainly due to uncertainty in the digital channel alignment. The decrease in velocity with increasing thermal damage is quite dramatic. The variation in velocity is on the order of $18 \%$ for the two extremes of temperature exposure. The correlation with extended temperatures is excellent and provides a method to assess thermal damage. Although the velocity showed a large change with thermal damage, the Barcol hardness numbers provided by McDonnell Douglas showed little variation over

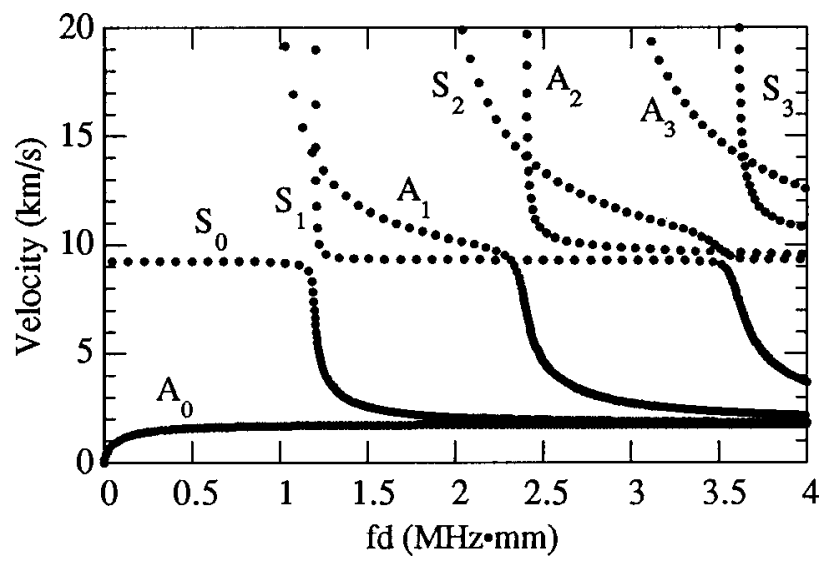

FIG. 9. Theoretical dispersion curves for an AS4/977-3 unidirectional composite.

this temperature range. Therefore, measurement of the Lamb wave velocity could provide a better technique to assess thermal damage than conventional methods.

A model calculation ${ }^{36,37}$ to determine the dispersion curves for this material system was computed using engineering constants supplied by McDonnell Douglas. The four standard engineering values $\left(E_{1}, E_{2}, G_{12}\right.$, and $\left.\nu_{12}\right)$ can be used to calculate the corresponding five elastic stiffness parameters $\left(c_{11}, c_{13}, c_{23}, c_{33}\right.$, and $\left.c_{55}\right)$ using various techniques. ${ }^{1}$ Since a value for $G_{23}$ was not available, the value of $c_{44}$ [where $c_{44}=\left(c_{22}-c_{23}\right) / 2$ ] must be estimated using common values for polymer matrix/graphite fiber composites. ${ }^{39}$ The value of $c_{44}$ typically ranges from 3.5 to 4.0 GPa, so a median value of $3.75 \mathrm{GPa}$ was chosen for the calculation. The density of the material was taken to be $1560 \mathrm{~kg} / \mathrm{m}^{3}$. A five layer model calculation of the dispersion curves for propagation in the fiber direction is shown in Fig. 9.

From the dispersion curve, one can see that the $S_{0}$ mode is almost completely nondispersive for the lower frequencythickness products. Also, the $S_{0}$ velocity in this region is around $9 \mathrm{~km} / \mathrm{s}$, which is exactly the velocity that was measured for the samples with little or no damage. Thus the model compares well with the experimentally determined velocity.

Theoretical dispersion curves were also calculated for AS4/977-3 using reduced stiffness constants. The effect of reducing different $c_{I J}$ 's by $20 \%$ on the low frequency, nondispersive region of the lowest order symmetric mode $(0.0$ $\leqslant f d \leqslant 1.0 \mathrm{MHz} \cdot \mathrm{mm}$ ) was investigated. The only parameter which altered the velocity of the $S_{0}$ mode in this region for propagation along the fibers is $c_{11}$.

Figure 10 shows a plot of the experimentally determined normalized velocity versus exposure temperature, where the normalized velocity is the velocity in the damaged sample divided by the velocity in an undamaged control sample. Also plotted is the normalized velocity of the $S_{0}$ mode with reduced values of $c_{11}$. Here, the normalized velocity is the velocity with $c_{11}$ reduced divided by the velocity with the full values of the elastic stiffness constants. Both velocities were taken at a constant frequency-thickness product of $0.5 \mathrm{MHz} \cdot \mathrm{mm}$ on the dispersion curve. Theoretical calcula- 


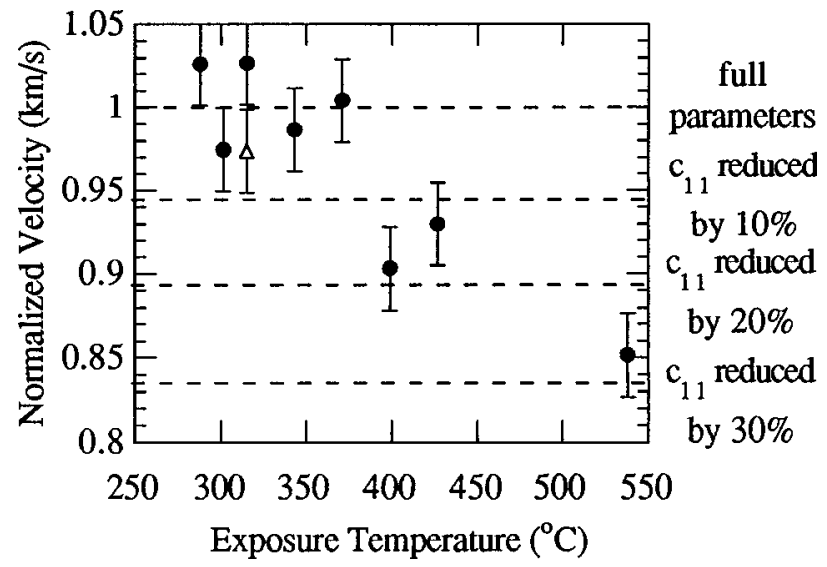

FIG. 10. Plot of the normalized $S_{0}$ velocity of samples exposed to different temperatures. The duration times are $3 \mathrm{~min}$ (solid circles) and $10 \mathrm{~min}$ (open triangle). Also shown (dashed lines) is the theoretically determined normalized $S_{0}$ velocity with reduced values of $c_{11}$, as described in the text.

tions with reduced values of $c_{11}$ show that a $25 \%-30 \%$ reduction in this parameter lowered velocities of the $S_{0}$ mode by $15 \%$. This value corresponds to the velocity reduction of the sample which saw an exposure temperature of $538^{\circ} \mathrm{C}$.

\section{LASER DAMAGE}

The three laser damaged samples examined were fabricated as quasi-isotropic plates with a stacking sequence of $[0 /+45 /-45 / 90]_{S}$. The fiber/matrix used on two of the samples was IM7/SP500 and the remaining sample was IM7/ PEEK. All three samples were irradiated at $100 \mathrm{~W} / \mathrm{cm}^{2}$. The IM7/SP500 samples were subjected to the laser source for durations of 0.5 and $1.0 \mathrm{~s}$. The IM7/PEEK sample was subjected to the laser source for $0.5 \mathrm{~s}$.

In this study, Lamb wave velocities were measured using the same technique described in the second fatigue study. The velocity of the lowest order symmetric Lamb mode was measured in the $0^{\circ}$ direction in both the undamaged and damaged regions of each of the samples. The velocities as well as the damage level for each of the different samples are shown in Table I. The listed velocities are the average of three measurements performed in each region. For each of the three measurements in a particular region, the values of the velocity varied less by less than $5 \%$ from the average.

As can be seen from the data, the velocity actually increases in the area which was subjected to the laser source. This is contrary to what was seen in the thermally damaged samples discussed earlier in this paper. The main difference between the laser damage and the thermal damage previously mentioned is that the laser subjects the sample to an extremely rapid thermal cycle. This type of heating could

TABLE I. Data for laser damaged samples.

\begin{tabular}{cccccc}
\hline \hline Sample & $\begin{array}{c}\text { Irradiation } \\
\left(\mathrm{W} / \mathrm{cm}^{2}\right)\end{array}$ & $\begin{array}{c}\text { Time } \\
(\mathrm{s})\end{array}$ & $\begin{array}{c}\text { Fluence } \\
\left(\mathrm{J} / \mathrm{cm}^{2}\right)\end{array}$ & $\begin{array}{c}\text { Undamaged } \\
\text { Vel. }(\mathrm{km} / \mathrm{s})\end{array}$ & $\begin{array}{c}\text { Damaged } \\
\text { Vel. }(\mathrm{km} / \mathrm{s})\end{array}$ \\
\hline IM7/SP500 & 100 & 0.5 & 50.0 & 6.01 & 6.10 \\
IM7/SP500 & 100 & 1.0 & 100.0 & 5.91 & 6.33 \\
IM7/PEEK & 100 & 0.5 & 50.0 & 6.50 & 6.56 \\
\hline \hline
\end{tabular}

actually cause embrittlement of the sample, which would serve to increase the velocity of the Lamb wave in that region.

\section{CONCLUSION}

The Lamb wave velocity measurements in this study were conducted at long wavelengths. This was done for several reasons. First, if the wavelength is large compared to the diameter of the fibers, composites can be treated as homogeneous. Second, only the lowest order modes propagate in the frequency range where the contact measurements were conducted. Finally, the effective elastic properties of the material can be measured because local defects, which scatter high frequency waves, will not be observed at long wavelengths.

Lamb waves offer a useful technique for characterizing many forms of damage in composite materials. Lamb wave velocity measurements are better than conventional ultrasonic measurement schemes (i.e., through-the-thickness measurements) because they can propagate over long distances and are sensitive to the desired in-plane elastic properties of the material. The propagation of Lamb waves depends on a variety of material properties: elastic stiffness constants, density, and thickness. As a material is damaged in some fashion, one or more of these material properties are altered. Since the Lamb wave velocity is directly related to these parameters, an effective tool exists to monitor damage in composites by measuring the velocity of these waves.

The Lamb wave velocity is a quantitative measurement and it has been shown by this work to be an effective tool in monitoring both fatigue and thermal damage in composites. Thus the Lamb wave method can be used to verify the integrity of a composite structure over the service life of a component. This is an important measurement for flight qualified composite materials that may have undergone some measure of nonvisible damage, which could seriously degrade the performance of the structure. Since the Lamb wave velocity depends on a variety of material properties, an ideal technique exists to monitor composites as damage is incurred. With the continued development of damage assessment techniques such as the Lamb wave method, the safety of such structures can be assured.

\section{ACKNOWLEDGMENTS}

The authors would like to thank John Masters of Lockheed Engineering and Sciences for providing the samples used in the fatigue studies. They would also like to thank Kevin Slattery of McDonnell Douglas Aerospace for providing the thermally damaged samples and Bill Laughlin of Physical Sciences, Inc. for providing the laser damaged samples. This work was supported in part by NASA Grant No. NAG-1-1063 and NASA Task Assignment No. NAS119656.

\footnotetext{
${ }^{1}$ I. M. Daniel and O. Ishai, Engineering Mechanics of Composite Materials (Oxford U. P., New York, 1994), pp. 3-76.

${ }^{2}$ D. Stover, senior editor, "Composites Use Increases on New Commercial Transports...," Advanced Composites 6, 30-40 (1991).
} 
${ }^{3}$ Composites News, "Boron/Epoxy System to Repair Entire B-1 Fleet," Advanced Composites 6, 18-20 (1991).

${ }^{4}$ T. K. O'Brien, M. Rigamonti, and C. Zanotti, “Tension fatigue analysis and life prediction for composite laminates," Int. J. Fatigue 11, 379-393 (1989).

${ }^{5}$ A. Charewicz and I. M. Daniel, "Damage mechanisms and accumulation in graphite/epoxy laminates," in Composite Materials: Fatigue and Fracture, ASTM STP 907, edited by H. T. Hahn (American Society for Testing and Materials, Philadelphia, 1986), pp. 274-297.

${ }^{6}$ S. L. Ogin, P. A. Smith, and P. W. R. Beaumont, "Matrix cracking and stiffness reduction during the fatigue of a (0/90)s GFRP laminate," Compos. Sci. Technol. 22, 23-31 (1985).

${ }^{7}$ S. L. Ogin, P. A. Smith, and P. W. R. Beaumont, "A stress intensity factor approach to the fatigue growth of transverse ply cracks," Compos. Sci. Technol. 24, 47-59 (1985).

${ }^{8}$ S. G. Lim and C. S. Hong, "Prediction of transverse cracking and stiffness reduction in cross-ply laminated composites," J. Compos. Mater. 23, 695-713 (1989).

${ }^{9}$ N. Laws and G. J. Dvorak, "Progressive transverse cracking in composite laminates," J. Compos. Mater. 22, 900-916 (1988).

${ }^{10}$ K. N. Street, A. J. Russell, and F. Bonsang, "Thermal damage effects on delamination toughness of a graphite/epoxy composite,' Compos. Sci. Technol. 32, 1-14 (1988)

${ }^{11} \mathrm{C}$. T. Herakovich and M. W. Hyer, "Damage-induced property changes in composites subjected to cyclic thermal loading," Eng. Fract. Mech. 25, 779-791 (1986).

${ }^{12}$ P. G. Ermer and S. Mall, "Damage mechanisms in a unidirectional metal matrix composite during thermal cycling," in American Society for Composites, Technical Conference, 5th, East Lansing, MI, June 12-14, 1990, Proceedings (A91-26701 10-4) (Technomic, Lancaster, PA, 1990), pp. $528-536$

${ }^{13}$ K. E. Stansfield and E. Pritchard, "Damage Generation and Healing During Composite Thermal Spikes," in Proceedings of the International SAMPE Technical Conference, 21st, Atlantic City, NJ, 25-28 Sept. 1989, pp. 120-129.

${ }^{14}$ M. J. Salkind, "Early Detection of Fatigue Damage in Composite Materials," AIAA Paper No. 75-772, AIAA/ASME/SAE 16th Structures, Structural Dynamics, and Materials Conference (Denver, Colorado, 27-29 May 1975), pp. 1-8.

${ }^{15}$ W. W. Stinchcomb, "Nondestructive evaluation of damage accumulation processes in composite laminates," Compos. Sci. Technol. 25, 103-118 (1986)

${ }^{16}$ I. M. Daniel, S.-C. Wooh, and J.-W. Lee, "Nondestructive evaluation of damage development in composite materials," in Elastic Waves and Ultrasonic Nondestructive Evaluation, edited by S. K. Datta, J. D. Achenbach, and Y. S. Rajapakse (Elsevier Science, Amsterdam, 1990), pp. 183189.

${ }^{17}$ V. Dayal and V. K. Kinra, "Ultrasonic NDE of Composites for Transverse Cracking," in Optical Methods in Composites; Proceedings of the SEM Fall Conference on Experimental Mechanics, Keystone, CO, 2-5 Nov. 1986 (A88-13876 03-24) (Society for Experimental Mechanics, Inc., Bethel, CT, 1986), pp. 17-22.

${ }^{18}$ B. Tang and E. G. Henneke II, "Lamb-wave monitoring of axial stiffness reduction of laminated composite plates," Mater. Eval. 47, 928-934 (1989).

${ }^{19}$ V. Dayal, V. Iyer, and V. K. Kinra, "Ultrasonic evaluation of microcracks in composites," in Advances in Fracture Research; Proceedings of the Seventh International Conference on Fracture (ICF7), Houston, TX, 20-24 Mar. 1989, Vol. 5 (A90-41276 18-39) (Pergamon, Oxford, 1989), pp. 3291-3300.

${ }^{20}$ M. R. Karim, A. K. Mal, and Y. Bar-Cohen, "Inversion of leaky Lamb wave data by simplex algorithm," J. Acoust. Soc. Am. 88, 482-491 (1990).

${ }^{21}$ A. K. Mal, M. R. Gorman, and W. H. Prosser, "Material characterization of composite laminates using low-frequency plate wave dispersion data," in Review of Progress in Quantitative Nondestructive Evaluation, edited by D. O. Thompson and D. E. Chimenti (Plenum, New York, 1992), Vol. 11, pp. 1451-1458.
${ }^{22}$ C. C. Habeger, R. W. Mann, and G. A. Baum, "Ultrasonic plate waves in paper,' Ultrasonics 17, 57-62 (1979).

${ }^{23}$ R. A. Kline and M. M. Doroudian, "Ultrasonic evaluation of adhesively bonded composites using guided waves," in Advances in Composite Materials and Structures (A91-23701 08-24) (American Society of Mechanical Engineers, New York, 1989), pp. 63-67.

${ }^{24}$ P. N. Dewen and P. Cawley, "An ultrasonic scanning technique for the quantitative determination of the cohesive properties of adhesive joints," in Review of Progress in Quantitative Nondestructive Evaluation, edited by D. O. Thompson and D. E. Chimenti (Plenum, New York, 1992), Vol. 11 , pp. $1253-1260$.

${ }^{25}$ M. J. S. Lowe and P. Cawley, "The applicability of plate wave techniques for the inspection of adhesive and diffusion bonded joints," J. Nondestruct. Eval. 13, 185-200 (1994)

${ }^{26}$ Keun J. Sun, "Application of guided acoustic waves to delamination detection," in Review of Progress in Quantitative Nondestructive Evaluation, edited by D. O. Thompson and D. E. Chimenti (Plenum, New York, 1992), Vol. 11, pp. 1213-1219.

${ }^{27} \mathrm{~N}$. Guo and P. Cawley, "The interaction of Lamb waves with delaminations in composite laminates," J. Acoust. Soc. Am. 94, 2240-2246 (1993).

${ }^{28}$ N. Guo and P. Cawley, "Lamb waves for the NDE of composite laminates," in Review of Progress in Quantitative Nondestructive Evaluation, edited by D. O. Thompson and D. E. Chimenti (Plenum, New York, 1992), Vol. 11, pp. 1443-1450.

${ }^{29}$ Y. Bar-Cohen and D. E. Chimenti, "NDE of defects in composites using leaky Lamb waves," in Symposium on Nondestructive Evaluation, 15th, San Antonio, TX, 23-25 April 1985, Proceedings (A86-47129 22-38) (Nondestructive Testing Information Analysis Center, San Antonio, TX, 1986), pp. 202-208.

${ }^{30}$ K. Balasubramaniam and J. L. Rose, "Guided Plate Wave Potential for Damage Analysis of Composite Materials," in Review of Progress in Quantitative Nondestructive Evaluation, edited by D. O. Thompson and D. E. Chimenti (Plenum, New York, 1990), Vol. 9, pp. 1505-1512.

${ }^{31}$ Y. J. Lee, B. T. Khuri-Yakub, and K. C. Saraswat, "Temperature measurements in rapid thermal processing using acoustic techniques," Rev. Sci. Instrum. 65, 974-976 (1994).

${ }^{32}$ J. Pei, F. Levent Degertekin, B. T. Khuri-Yakub, and K. C. Sarawsat, "In situ thin film thickness measurement with acoustic Lamb waves,' Appl. Phys. Lett. 66, 2177-2179 (1995).

${ }^{33}$ J. J. Ditri, A. Pilarski, B. Pavlakovic, and J. L. Rose, "Generation of guided waves in a plate by axisymmetric normal surface loading," in Review of Progress in Quantitative Nondestructive Evaluation, edited by D. O. Thompson and D. E. Chimenti (Plenum, New York, 1994), Vol. 13, pp. 133-140.

${ }^{34} \mathrm{~W}$. H. Prosser, "The propagation of characteristics of the plate modes of acoustic emission waves in thin aluminum plates and thin graphite/epoxy composite plates and tubes," Ph.D. thesis, The Johns Hopkins University, 1991.

${ }^{35} \mathrm{~K}$. Balasubramaniam and J. L. Rose, "Physically based dispersion curve feature analysis in the NDE of composites," Res. Nondestruct. Eval. 3, 41-67 (1991).

${ }^{36}$ S. B. Dong and K. H. Huang, "Edge vibrations in laminated composite plates," J. Appl. Mech. 52, 433-438 (1985).

${ }^{37}$ S. K. Datta, A. H. Shah, and W. Karunasena, "Wave propagation in composite media and material characterization," in Elastic Waves and Ultrasonic Nondestructive Evaluation, edited by S. K. Datta, J. D. Achenbach, and Y. S. Rajapakse (Elsevier Science, Amsterdam, 1990), pp. 159167.

${ }^{38}$ M. Caslini, C. Zanotti, and T. K. O'Brien, "Study of matrix cracking and delamination in glass/epoxy laminates," J. Composites Technol. Res. 9, 121-130 (1987)

${ }^{39}$ W. H. Prosser, "Ultrasonic characterization of the nonlinear elastic properties of unidirectional graphite/epoxy composites," NASA Contractor Report 4100, NASA Langley Research Center, October, 1987, p. 65. 